\title{
PRIMER REGISTRO DEL PALEOCENO EN EL URUGUAY: PALEOSUELOS CALCAREOS FOSILIFEROS EN LA CUENCA DE SANTA LUCIA
}

\author{
SERGIO MARTÍNEZ ; GERARDO VEROSLAVSKY \\ \& MARIANO VERDE*
}

\begin{abstract}
PALEOCENE FOSSILASSOCIATIONSIN CALCAREOUS PALEOSOLS FROM SANTA LUCIA BASIN (URUGUAY) For the first time, the Paleocene is recorded in Uruguay, developed at the central area of Santa Lucia Basin. It is represented by non depositional events, recognized as carbonatic paleosols, containing a characteristic fossil record. Up to date these carbonate deposits were included in the deposits known as "Calizas del Queguay" (Queguay Limestones), and considered as belonging to Mercedes Formation (Upper Cretaceous), interpreted as lacustrine facies laterally associated with sandstone and conglomérate fluvial facies. However, researches conducted by the authors have shown that these deposits are the product of calcretization processes that affected the Mercedes Formation clastic sedimentites. The calcareous, fossiliferous paleosols were developed overimposed at the top of the groundwater calcretes. The fossils found are: pulmonate gastropods (Eoborus charruanus), endocarps of Celtis santosi, rizoliths, and bee nests (Celliforma spp.). This association is typical of paleosols and the age of all the fossils is Tertiary. The gastropods have very similar representatives at the Paleocene of the São José de Itaboraí Basin (Rio de Janeiro, Brazil), being the Uruguayan and Brazilian representatives of Eoborus the only species known of this genus. The Celtis santosi endocarps are present only at these two basins. Then, a Paleocene age is postulated for the paleosols and for the event of calcretization, developed under a warm and arid environment.
\end{abstract}

Key words: Paleocene, gastropods, ichnofossils, seeds, Queguay Limestones, Unuguay

\begin{abstract}
RESUMEN Por primera vez se reconocen registros del Paleoceno en el Uruguay, los que se desarrollan en la región central de la Cuenca de Santa Lucía. Los mismos constituyen eventos no depositacionales, que son identificados como niveles de paleosuelos carbonáticos portadores de un característico registro de flora y fauna fósil . Hasta el momento, estos depósitos fueron incluidos en lo que genéricamente se denomina como "Calizas del Queguay", considerados como pertenecientes a la Formación Mercedes (Cretácico Superior) e interpretadas como facies lacustres asociadas lateralmente a facies de areniscas y conglomerados fluviales. Los trabajos realizados permiten descartar ese origen para los depósitos carbonáticos y se interpretan como producto de procesos de calcretización que afectaron a las areniscas y conglomerados de la Formación Mercedes. Hacia el tope y sobreimpuestos a las calcretas de aguas subterráneas se desarrollan los paleosuelos calcáreos fosilíferos. Los fósiles hallados corresponden a gastrópodos (Eoborus charruanus), endocarpos de Celtis santosi, rizolitos, y nidos de abejas (Celliforma ispp.). Todos los fósiles corresponden al Terciario, pero además se destaca que los gastrópodos y los endocarpos tienen representantes muy similares en el Paleoceno de la Cuenca de Sao José de Itaborá (Rio de Janeiro, Brasil). En el caso de Eoborus se trata de los únicos representantes del género, en tanto que Celtis santosi se halla úúnicamente en estas cuencas. Por lo tanto, se postula una edad Paleocena para los paleosuelos fosilíferos, y consecuentemente también para el proceso de calcretización, desarrollados bajo condiciones áridas y cálidas.
\end{abstract}

Palabras clave: Paleoceno, gastrópodos, icnofósiles, frutos, Calizas del Queguay, Uruguay
INTRODUCCIÓN Las llamadas "Calizas del Queguay" (término acuñado por Lambert 1939, pero descrip- tas en la región que les da nombre en 1940) comprenden
El material fosilífero estudiado se encuentra depositado en la colección del Departamento de Paleontología de la Facultad de Ciencias (FCDP), Montevideo. depósitos carbonáticos que afloran en el litoral oeste y centrosur del Uruguay, los que han sido interpretados y formalizados en la terminología estratigráfica de diversas maneras (ver Veroslavsky et al., en prensa).

Los fósiles atribuidos a las "Calizas del Queguay" nunca fueron posicionados precisamente dentro de la unidad. En realidad, como se verá más adelante, ellos solamente se encuentran en los niveles superiores, asociados a paleosuelos sobreimpuestos a calcretas de aguas subterráneas. Estas últimas han sido descriptas en Veroslavsky et al. (1997), realizando una reinterpretación genética que obliga a una total revalorización de la edad de las "Calizas del Queguay", en tanto la carbonatación representa un episodio temporal sin correlato sedimentario.

En este trabajo se describen los fósiles de las "Calizas del Queguay" presentes en la Cuenca de Santa Lucía, demostrándose su edad Paleocena y haciendo referencia a su paleoambiente. Se trata de las primeras evidencias de eventos y procesos ocurridos durante el Paleoceno en el Uruguay.

La ubicación geográfica de los afloramientos estudiados se puede observar en la Fig. 1.

\section{ANTECEDENTES Y MARCO GEOLÓGICO Las}

"Calizas del Queguay" fueron tradicionalmente interpretadas como depósitos lacustres asociados a los estadios evolutivos finales de la sedimentación cretácica (Bossi et al. 1975, Preciozzi et al. 1985, Morales \& Ford 1988, de Santa Ana et al. 1993) asociados a la Fm. Mercedes (areniscas gruesas, blancas a rojizas, mal seleccionadas, con intercalaciones de lentes conglomerádicos y pelíticos, correspondientes a depósitos de abanicos aluviales y ríos entrelazados). Recientemente, este concepto ha sido objeto de revisión y dichos depósitos son considerados como groundwater calcretes (Veroslavsky et al, 1997), cuya formación afectó principalmente a las sedimentitas neocretácicas.

El criterio equívoco de considerar a las calizas como un evento depositacional lacustre, trajo consigo grandes controversias en relación a la edad y el posicionamiento estratigráfico de éstos depósitos (ver Tabla 1). Las relaciones de intercalación entre los lentes calcáreos y las sedimentitas clásticas de la Formación Mercedes observadas por Bossi et al. (1975) y consecuentemente, su supuesto sincronismo,

* Departamento de Paleontología y ** Departamento de Geología, Facultad de Ciencias, T. Narvaja 1674, 11200 Montevideo, Uruguay. - e-mail: smart@fcien.edu.uy,FAX: (5982)409973 


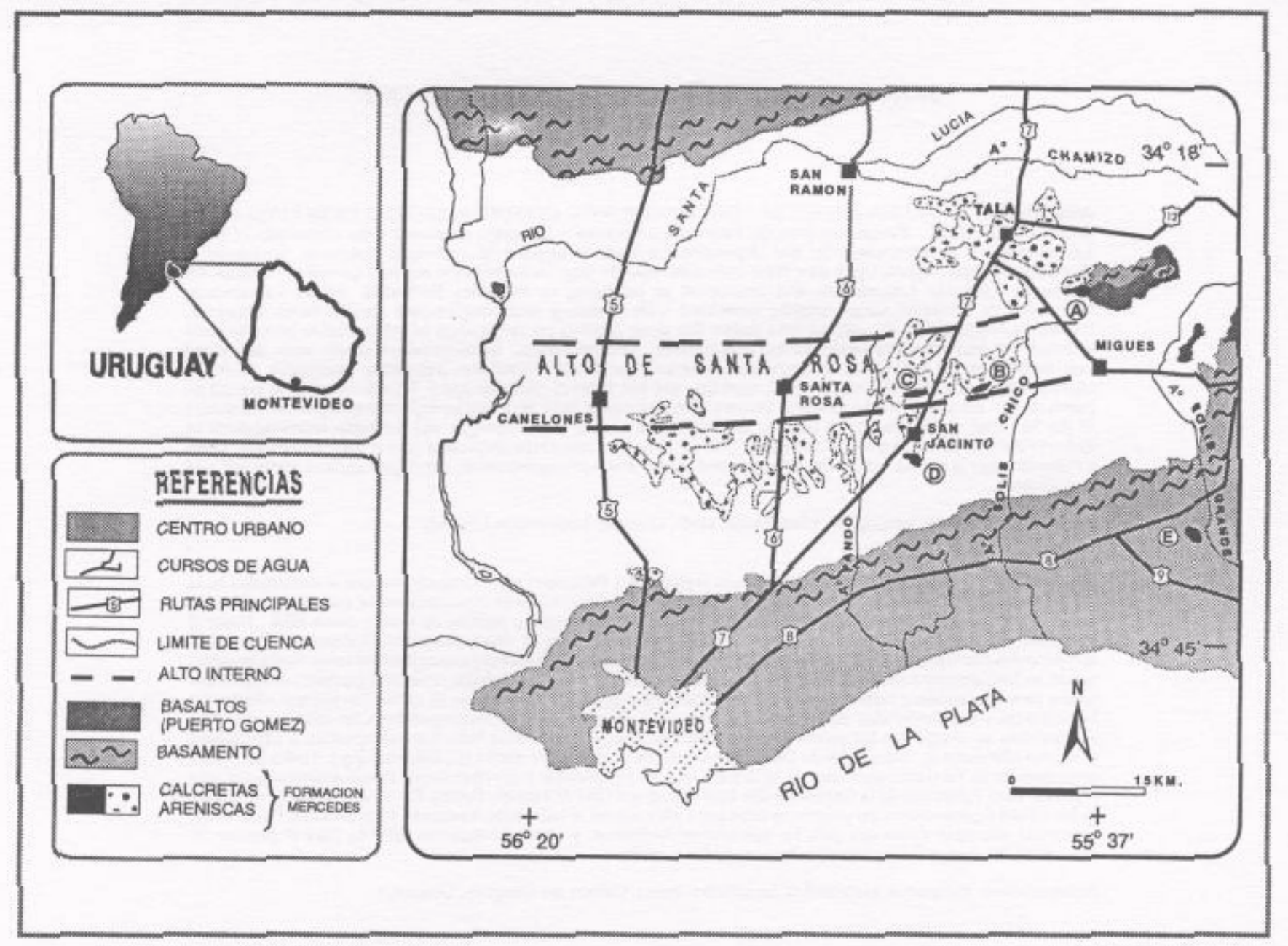

Figura 1 - Ubicación geográfica de los afloramientos de calcretas en la Cuenca de Santa Lucía. A: Sauce Solo. B: Piedra Sola. C: Arroyo Cochengo. D: Sur de San Jacinto. E: SW de Solís de Mataojo.

Figure 1 - Geographic location of the calcrete-bearing outcrops at the Santa Lucia Basin. A: Sauce Solo. B: Piedra Sola. C: Cochengo Creek. D: South of San Jacinto. E: SW from Solís de Mataojo.

condujo a considerarlas cretácicas. Este criterio prevaleció frente a los argumentos paleontológicos que situaban las calizas en el Terciario (Frenguelli 1930, Walther 1931, Caorsi \& Goñi 1958, Goso \& Bossi 1966).

Los fósiles atribuidos a las "Calizas del Queguay" nunca fueron posicionados precisamente. Uno de los hechos significativos del presente trabajo es señalar que los fósiles se hallan siempre en horizontes calcáreos algo arenosos, ocasionalmente gravillosos, con espesores de orden métrico y expresiva tabularidad y continuidad lateral, a veces algo silicificados, a los que se interpreta como paleosuelos. Estos paleosuelos se desarrollan directamente sobre las calcretas de aguas subterráneas, como por ejemplo en la región de Sauce Solo (Fig. 2), sobre las sedimentitas neocretácicas como los del Arroyo Cochengo, o sobre el basamento cristalino en la región al SW de Solís de Mataojo.

Las principales evidencias que sustentan esta interpretación son : a) el desarrollo de un nivel continuo, de espesor métrico y expresiva continuidad lateral; b) microscópicamente, está constituido por glébulas isótropas, a veces ar-

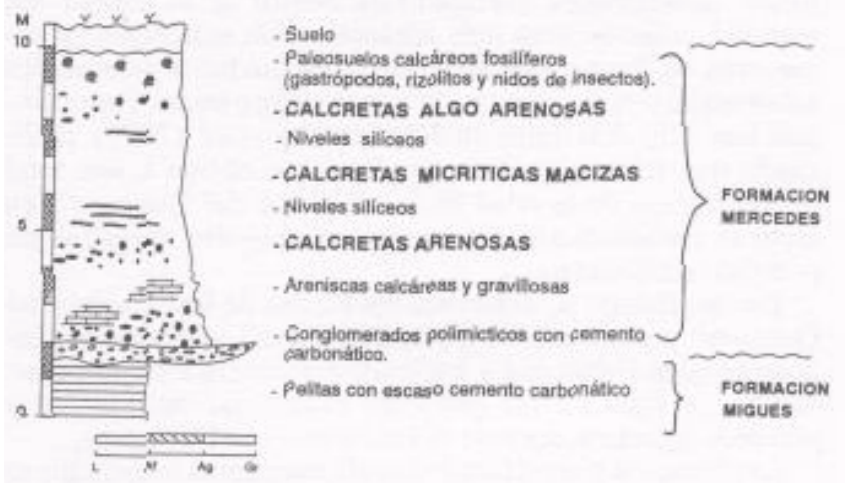

Figura 2 - Sección estratigráfica de la cantera de Sauce Solo (frente norte).

Figure 2 - Stratigraphic column of the Suce Solo quarry (facing north). 
Tabla 1 - Sumario de las diferentes opiniones respecto a la edad de las "Calizas del Queguay" Table 1 - Different views about the age of the "Queguay Limestones".

\begin{tabular}{|l|c|c|c|c|c|c|c|}
\hline & Cretícico & Terciario & Paleógeno & Palcoceno & Eoceno? & Oligoceno & Mio-Plioceno \\
\hline Frenguelli (1930) & & $\mathrm{X}$ & & & & & \\
\hline Walther (1931) & & & & & & & $\mathrm{X}$ \\
\hline Lambert (1939) & & $\mathrm{X}$ & & & & & \\
\hline Lambert (1940) & $\mathrm{X}$ & $\mathrm{X}$ & & & & $\mathrm{X}$ & \\
\hline Serra (1945) & & & & & & $\mathrm{X}$ & \\
\hline Jones (1956) & & & $\mathrm{X}$ & & & & \\
\hline Goso y Bossi (1966) & $\mathrm{X}$ & & & & & & \\
\hline Bossi ef al. (1975) & & & & & $\mathrm{X}$ & & \\
\hline Mones (1979) & $\mathrm{X}$ & & $\mathrm{X}$ & & & & \\
\hline Sprechumann et al.(1981) & $\mathrm{X}$ & & & & & & \\
\hline Bossi y Navarro (9991) & & & & $\mathrm{X}$ & & & \\
\hline este trabajo & & & & & \\
\hline
\end{tabular}

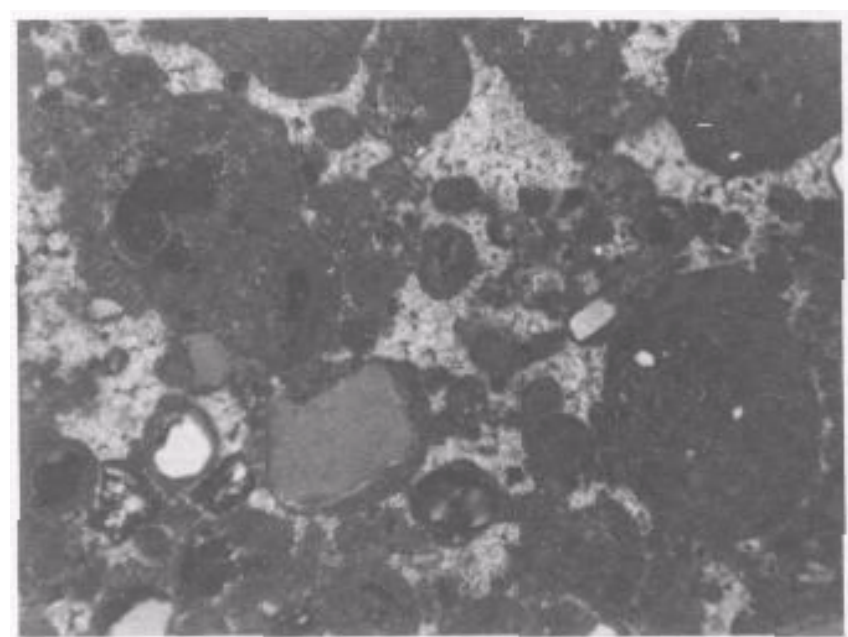

Figura 3- Fotomicrografia de la calcreta pedogénica correspondiente al nivel superior del perfil de la figura 2. Se observan clastos de cuarzo reemplazados por material micrítico hacia los bordes y los espacios intergranulares rellenos por material esparitico. (escala $x 35$, nicoles cruzados).

Figure 3 - Pedogenic calórete Photomicrograph from the upper section of the column shown in Figure 2. The external zones of quartz grains have been replaced by micrite. The intergranular spaces have sparitic material (scale $\mathrm{x}$ 35 , cross-polarized light).

cillosas, fracción clástica cuarzosa cementada por varias generaciones de calcita (Figs. 3 y 4); c) presencia de fósiles característicos de paleosuelos (Retallack 1990): gastrópodos terrestres, rizolitos, frutos, y nidos de insectos excavados y revestidos.

No han sido encontradas evidencias de campo que permitan definir un hiatus entre las calcretas y los paleosuelos calcáreos fosilíferos. Por lo tanto, se los considera desarrollados bajo un mismo evento, penecontemporáneo, configurando un perfil de carbonatación completo como el propuesto por Carlisle (1983). De esta forma, hacia la parte superior actúan los procesos edáficos $\mathrm{y}$, en profundidad, son predominantes gradualmente los procesos de acumulación de carbonates por fluctuación del nivel freático. De acuerdo con Goudie (1983) y Wright \& Tucker (1991) las condiciones climáticas para la generación de calcretas pedogenéticas y/o subterráneas son áridas y cálidas, con alta tasa de evapotranspiración.

Sólo uno de los puntos relevados (Sauce Solo, Figs. 1 y 2) proporcionó fósiles, encontrándose en la colección del Dpto. de Paleontología de la Facultad de Ciencias gastrópodos provenientes de otra (cantera de Arroyo Cochengo, Fig. 1), donde personalmente no los hallamos. Por otra parte la exposición de la primera localidad es muy superior, por lo que las consideraciones vertidas en este artículo se refieren especialmente a ella.

La presencia de fósiles en la cantera de Sauce Solo es conocida desde Frenguelli (1930), aunque este autor la ubicó incorrectamente respecto a la ciudad de Migues. Jones (1956) corrigió su ubicación

Los fósiles citados por Frenguelli (1930) son: raíces, celdas de véspidos, aquenios, oogonios de Charáceas, células epidérmicas de gramíneas, Borus charruanus (Gastropoda), y un gastrópodo raro y mal conservado. De ellos, no hemos hallado oogonios, lo que es consistente con la génesis propuesta más adelante.

El mineral que se encuentra formando los restos fósiles es predominantemente calcedonia (ver por ejemplo la Fig. 4), la que ha reemplazado a los constituyentes originales.

\section{PALEONTOLOGÍA SISTEMÁTICA}

Clase Dicotyledoneas Jussieu, 1789

Familia Ulmaceae Mirbel, 1815

Género Celtis Linné, 1753

Celtis santosi (Magalhães, 1950)

(Fig. 5a-b)

Acrocarpus santosi Magalhães, 1950 : 42, fig.

Celtis santosi - Beurlen \& Sommer, 1954: 16-18, est. I, figs. 1-2, 4-6; est. II, figs. 1-2, 4-6.

Celtis santosi - Palma \& Brito, 1974: 398.

M a ter i a 1: FCDP 2735 al 2742.

Diagnos is: Endocarpos esférico-ovoides a piriformes, redondeados en la base y puntiagudos en el ápice. Presentan una carena media, de base a ápice. Presenta rugosidades irregulares de elevación variable que forman un reticulado superficial. 


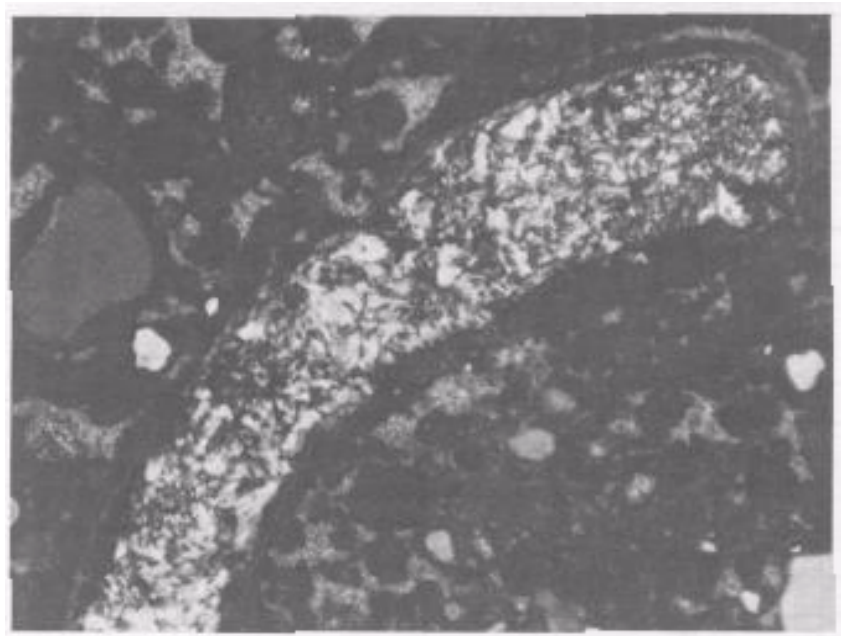

Figura 4 - Fotomicrografía de de la calcreta pedogénica donde se observa la concha de un gastrópodo pulmonado que fue reemplazada por calcedonia fibrosa (escala $x 35$, nicoles cruzados).

Figure 4 - Pedogenic calórete Photomicrograph showing the section of a pulmonate gastropod shell replaced by fibrous calcedony. (scale x 35, cross-polarized light).

Dimensiones (en mm):

$\begin{array}{lcl} & \text { largo } & \text { ancho } \\ \text { FCDP 2735 } & 3,9 & 3,0 \\ \text { FCDP 2736 } & 5,9 & 4,3 \\ \text { FCDP 2738 } & 5,9 & 4,9 \\ \text { FCDP 2740 } & 5,0 & 3,8 \\ \text { FCDP 2741 } & 4,5 & 3,4 \\ \text { FCDP 2742 } & 5,1 & 4,4\end{array}$

Comentários: El material consiste en endocarpos de los frutos de este género, perfectamente asimilables en morfología y dimensiones a Celtis santosi (Magalhães) hallada en el Paleoceno de la Cuenca de Sao José de Itaboraí (Estado de Rio de Janeiro, Brasil).

Frenguelli (1930) menciona la presencia de "aquenios" en el mismo afloramiento, sin descripción ni figuras. Consideramos que se trata del mismo tipo de fósiles que tratamos en esta sección.

Clase Gastropoda Cuvier, 1797

Familia Strophocheilidae Spix, 1827

Género Eoborus Klappenbach \& Olazarri, 1970

Eoborus charruanus (Frenguelli 1930)

(Fig. 5c-f)

Borus charruanus (Doello Jurado in litt.) in Frenguelli, 1930: 24-25, figs. 5-6.

Bulimus archiplatae (Ihering) in Walther, 1931: 35. (nomen nuduiri)

Borus charruanus - Lambert, 194166.

Bulimus archiplatae,-Lambcrt, 1941: 66.

Borus charruanus - Méndez Alzóla, 1944: 221.

Strophocheilus (Strophocheilus) atavus Bequaert, 1948: $47-$ 48 , pl. 8, fig. 3.

Borus (Strophocheilus) charruanus -Jones, 1956: 57.

Borus (Strophocheilus) charruanus -Caorsi \& Goñi, 1958: 56.

Strophocheilus (Strophocheilus) charruanus -Klappenbach \& Olazarri; 1966: 234-235, figs. 1-2.

Strophocheilus charruanus_Parodiz, 1969: 172-173, pl. 18, fig. 2 .

\section{Strophocheilus charruanus - Figueiras \& Broggi,} 1969: 341-343.

Eoborus charruanus - Klappenbach \& Olazarri, 1970: 180-181.

Eoborus charruanus - Klappenbach \& Olazarri, 1986:221.

M a te r i a 1: FCDP 2337 (lote de 4 ejemplares), FCDP 2684 al 2699, FCDP 2701 al

2709, FCDP 2711 al 2717.

D i a g n o s i s : Conchilla mediana, oval-oblonga. Espira cónica, algo corta. Ápice romo. 5-5 1/2 vueltas convexas. Vueltas nepiónicas lisas, el resto de las vueltas con estrías axiales gruesas y subparalelas. Ultima vuelta algo aplastada en sentido dorso-ventral. Sutura bien marcada, aunque no profunda. Columela lisa, labio externo reflejado. Ombligo grande. Abertura grande, ovoide. Callo parietal grande $\mathrm{D}$ i $\mathrm{m}$ e $\mathrm{n} \mathrm{s}$ i o n e s (en $\mathrm{mm}$ ): las dimensiones se brindan a modo ilustrativo, ya que la mayoría de los ejemplares presenta algún borde roto.

\begin{tabular}{lrrrrr} 
& alto & \multicolumn{2}{c}{$\begin{array}{c}\text { diám. max } \\
\text { diám. max } \\
\text { ventral }\end{array}$} & $\begin{array}{r}\text { largo } \\
\text { lateral }\end{array}$ & $\begin{array}{c}\text { ancho } \\
\text { abertura }\end{array}$ \\
FCDP2711 & 44,5 & 24,4 & 27,2 & - & - \\
FCDP2685 & 45,0 & 24,8 & 26,0 & - & - \\
FCDP2704 & 43,0 & 23,7 & 24,1 & 23,6 & 16,7 \\
FCDP2708 & 45,3 & 23,4 & - & - & -
\end{tabular}

Comentarios: La sinonimia refleja claramente los avalares nomenclaturales por los que ha pasado la especie. Debe agregarse que Klappenbach \& Olazarri (1966) consideraron con dudas como sinónimo a Strophocheilus oblongus Mull. var. crassa d'Orb. ( referido por Ihering 1897) = Strophocheilus oblongus crassus Albers ( según Ihering 1907 y 1914). Posteriormente Klappenbach \& Olazarri (1970) se refieren a ese material como entidad independiente.

De acuerdo con Klappenbach \& Olazarri $(1970,1986)$ el género Eoborus contendría por el momento con certeza solamente otras dos especies: 1) E. sanctijosephi (Maury), proveniente de la Cuenca de Sao José de Itaboraí (Estado de Rio de Janeiro, Brasil). Autores que han estudiado la cuenca de Sao José de Itaboraí han aceptado este criterio al referirse a los fósiles brasileros de esa manera; 2) E. berroi Klappenbach \& Olazarri, de origen estratigráfico incierto, pero a juzgar por las localidades que brindan los autores y por su forma de preservación podría provenir de otros afloramientos de las "Calizas del Queguay". Otras especies relacionadas con dudas (fundamentalmente por limitaciones del material fosilífero) son Strophocheilus chubutensis Ihering y S. hauthali Ihering, del Terciario de Argentina.

$$
\begin{gathered}
\text { Icnofósiles } \\
\text { Celliforma Brown, } 1934 \\
\text { Celliforma isp.A }
\end{gathered}
$$

(Fig. 53 , g)

"nidos de véspidos " - Frenguelli, 1930: 24-25; 1938a: 93-

$$
\text { 95, lám. VII, figs. 9-11; 1938b: }
$$$$
352 \text {, fig.3. }
$$

Material: FCDP 2728, 2731, 2732, 2733, 2734.

$\mathrm{D}$ i a $\mathrm{g} \mathrm{n}$ o s i s : Celdillas en forma de clava de paredes lisas, con cuello bien marcado. No se observa bioglifo.

$\mathrm{D}$ i m e n s i o n e s : longitud $11 \mathrm{~mm}$, diámetro máximo 6,5 $\mathrm{mm}$.

Com e n t a ri o s: Una de las muestras estudiadas contiene tres celdillas ubicadas paralelamente, en forma horizontal, una encima de la otra, separadas por $1,5 \mathrm{~mm}$ de distancia. Las tres se orientan de la misma forma, lo que hace pensar que convergen a un túnel de salida común, el cual no se observa. Por 


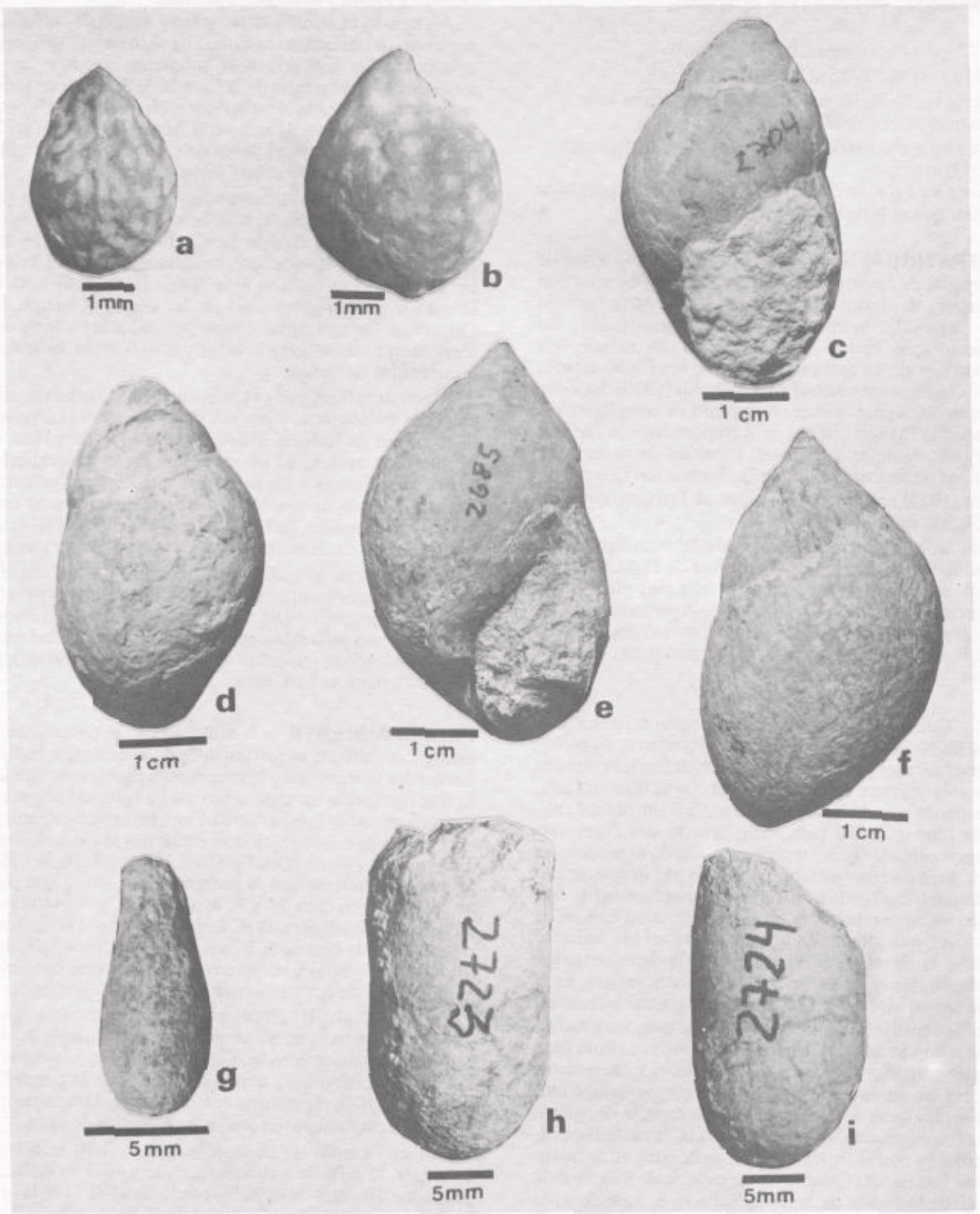

Figura 5 - Fósiles colectados en la Cantera de Sauce Solo, en unpaleosuelo de las "Calizas del Queguay", Paleoceno, a. Celtis nova C. santosi (Magalhães 1950), FCDP 2736. b. Celtis nova C. santosi (Magalhães 1950), FCDP 2740. c. Eoborus charruanus (Frenguelli 1930), vista ventral. FCDP2704. d. Eoborus charruanus (Frenguelli 1930), vista dorsal. FCDP 2704. e. Eoborus charruanus (Frenguelli 1930), vista ventral. FCDP 2685. f. Eoborus charruanus (Frenguelli 1930), vista dorsal. FCDP 2685. g. Celliforma isp. A, FCDP 2732. h. Celliforma isp. B. FCDP 2723. i. Celliforma isp. B. FCDP 2724.

Figure 5 - Fossils from Sauce Solo Quarry, collected from a "Queguay Limestones" paleosol. Paleocene. a. Celtis C. santosi (Magalhães 1950), FCDP 2736. b. Celtis C. santosi (Magalhães 1950), FCDP 2740. c. Eoborus charruanus (Frenguelli 1930), ventral view. FCDP2704. d. Eoborus charruanus (Frenguelli 1930), dorsal view. FCDP 2704. e. Eoborus charruanus (Frenguelli, 1930), ventral view. FCDP 2685. f. Eoborus charruanus (Frenguelli 1930), dorsal view. FCDP 2685. g. Celliforma isp. A, FCDP 2732. h. Celliforma isp. B. FCDP 2723. i. Celliforma isp. B. FCDP 2724. 
lo tanto, podría estar presente más de una icnoespecie, una de formas solitarias y otra de formas agrupadas.

\section{Celliforma isp.B (Fig. 5h-i)}

Material: FCDP 2726 al 2729.

D i a g n o s is : Celdillas subcilíndricas de paredes lisas, con un extremo redondeado.

D i m e n s i o n e s : longitud $20 \mathrm{~mm}$ (estimado), diámetro máximo $9,0 \mathrm{~mm}$.

Co m e n t a ri o s: la diferente morfología de las celdillas hace pensar que se trate de otra icnoespecie.

BIOESTRATIGRAFIA Francis (1975) crea la Zona de Agolpamiento de Eoborus charruanus, con perfil y área tipo en la Cantera de Sauce Solo, de donde proviene también nuestro material, aunque la somera descripción del afloramiento que realiza este autor, es incorrecta (ver Veroslavsky et al., en prensa). El resto de los fósiles citados para esta Zona son los mismos de Frenguelli (1930). La Zona de Agrupamiento de Eoborus charruanus es correlacionada por Francis (1975) con la Zona de Agrupamiento de Taphius waltheri (=Biomphalaria waltheri) (también de su autoría), que contiene otros gastrópodos de la "Formación Queguay".

Mones (1979) en una síntesis sobre el Terciario del Uruguay, acepta el esquema anterior.

En este trabajo no realizaremos nuevas consideraciones formales, aunque es claro que el esquema de Francis (1975) necesita de una profunda revisión. Sí resulta muy plausible la idea de la correlación de ambas "Zonas de Agrupamiento", ya que a pesar de contener faunas diferentes, en primera instancia se habrían desarrollado en condiciones geológicas globales similares.

EDAD Como fue expresado en la sección referente a la geología, los fósiles fueron encontrados, y vivieron, en paleosuelos desarrollados penecontemporáneamente a las calcretas de aguas subterráneas. Es decir, la edad que se discutirá aquí no corresponde a la de los sedimentos, sino aquella del momento de formación del paleosuelo. Por lo tanto, estamos tratando con unidades geocronológicas (cuándo se produjo un episodio), pero no cronoestratigráficas (no hay depositación) ni litoestratigráficas (no hemos definido alguna unidad de este tipo). Este enfoque representa una gran diferencia con todos los anteriores, que intentaban ubicar la edad de las "calizas" tal y como la de una unidad sedimentaria depositacional, utilizando siempre criterios estratigráficos tradicionales. En la tabla 1 se muestra las edades asignadas por autores anteriores.

Si bien los fósiles hallados no son de una gran variedad de especies, sí son lo suficientemente significativos como para argumentar respecto a su antigüedad. Eoborus charruanus pertenece a un género extinguido, con representantes únicamente en Uruguay y en Brasil. La otra especie uruguaya, como ya fue expresado, es de procedencia estratigráfica incierta pero muy probablemente provenga de otros afloramientos de las "Calizas del Queguay". La especie de Sao José de Itaboraí (Estado de Rio de Janeiro, Brasil) - E. sanctijosephi - es notablemente similar y afín a la que estamos tratando. El resto de las especies de Strophocheilidae exclusivamente fósiles han sido halladas en Argentina y -más allá de precisiones que no podemos realizar- las evidencias existentes indican que son Paleógenas (ver Parodiz, 1949, 1969).

La presencia de Celtis santosi (Magalhaes) relaciona también fuertemente esta asociación con la hallada en la Cuenca de Sao José de Itaboraí, ya que hasta el momento se conocía solo en ella (B curien \& Sommer 1954). El género es conocido por su polen con certeza desde el Eoceno, aunque podría encontrarse desde el Turoniano (Müller 1981). Otras referencias a endocarpos de Celtis que hemos hallado, son para el
Eoceno Inferior (Beherensmeyer et al. 1995) y Oligoceno (Retallak 1984a y b) de Norteamérica.

Aunque su ubicación estratigráfíca dentro de la Cuenca, y por ende su ubicación temporal, ha sido objeto de distintas opiniones, los trabajos más modernos indican que los moluscos y los vegetales de la Cuenca de Sao José de Itaboraí se encuentran ambos en depósitos asignados al Paleoceno temprano (Rodrigues Francisco \& Souza Cunha 1978, Klein et al. 1985). Análisis del polen de esos niveles han indicado una edad Paleocena-Eocena (Lima \& Cunha 1986).

La presencia de Celliforma no es indicativa de un rango temporal preciso, pero no contradice el resto de las inferencias, ya que según Ekdale et al. (1984) los representantes fósiles del orden Hymenoptera se conocen desde el Triásico, las avispas no se vuelven abundantes como fósiles hasta el Cretácico, y el registro fósil de las abejas comienza en el Oligoceno-Eoceno tardío. Como resultado, la mayoría de los icnogéneros atribuidos a avispas y abejas están incluidos en las unidades terciarias.

El conjunto fosilífero y el paleosuelo que lo contiene, serían entonces Paleógenos, y por sus relaciones con la asociación de Sao José de Itaboraí, Paleocenos. Dado que no han sido encontradas evidencias de un hiatus entre las calcretas de aguas subterráneas y los paleosuelos calcáreos fosilíferos, y que por lo tanto, se considera son parte de un mismo evento penecontemporáneo, ésta sería también la edad del proceso de calcretización descripto por Veroslavsky et al. (en prensa).

Existen otras asociaciones fósiles en las "Calizas del Queguay" que afloran en el litoral oeste del Uruguay (ver por ejemplo Frenguelli 1930, Lambert 1940, Bossi \& Navarro, 1991), las que probablemente tengan la misma edad que las consideradas aquí, aunque se necesitarán nuevos estudios para afirmarlo más rotundamente.

PALEOAMBIENTE A este respecto se observa una coincidencia entre los requerimientos de la biota que habitó en o sobre los paleosuelos y los necesarios para la calcretización, lo que representa un argumento más a favor de la penecontemporaneidad de estos eventos. Entre los requerimientos para un proceso de calcretización se encuentra la existencia de un clima cálido y árido (Goudie 1983, Wright \& Tucker 1991), lo que es coherente con la presencia de Celtis, que cuenta actualmente con entre 70 y 80 especies que viven en regiones templadas y subtropicales (Cabrera \& Zardini 1978), y con la observación de Genise \& Bown (1994), quienes indican que los nidos de avispas y abejas fosoriales muestran condiciones climáticas de aridez y ambientes de escasa vegetación.

En cuanto al pH, Retallack (1984a) menciona que los gastrópodos terrestres no se encuentran en suelos ácidos o neutrales, pero son comunes en los calcáreos, alcalinos $(\mathrm{pH}$ 7,5 - 8). Esto se debe a que la disolución de la conchilla en suelos ácidos es rápida una vez destruido el periostraco, un año aproximadamente en suelos no cargados de agua.

Los endocarpos de la especie actual Celtis occidentalis contienen $25-64 \%$ de carbonato de calcio y $2-7 \%$ de sílice por peso seco del fruto total, atribuyendo Retallak (1984a) a este hecho la preservación diferencial de Celtis hatcheri Chaney, 1925 en paleosouelos calcáreos del Oligoceno de Norte América. El hecho de haberse encontrado solamente este género de vegetales en Sauce Solo, no obedecería entonces necesariamente a una dominancia en su comunidad, sino más probablemente a un desvío tafonómico. La mineralización del endocarpo es una adaptación para resistir el pasaje a través del tracto digestivo de los herbívoros.

Las celdas fósiles de abejas son indicadores de áreas relativamente abiertas. Es muy probable que la vegetación dominante haya sido de angiospermas, fuente de polen para las abejas. Según Roubik (in Thackray 1994) las abejas que 
anidan en suelos prefieren sustratos de superficie firme en combinación con materiales más blandos en profundidad.

CONCLUSIONES Los fósiles atribuidos a las "Calizas del Queguay" en la Cuenca de Santa Lucía se encuentran ubicados en paleosuelos calcáreos, desarrollados penecontemporáneamente al proceso de calcretización que afectara principalmente a los sedimentos neocretácicos.

La asociación fósil guarda estrechas relaciones con la que se encuentra en la cuenca de São José de Itaboraí (Rio de Janeiro, Brasil).
Estos procesos se desarrollaron en el Paleoceno, bajo un régimen climático árido y cálido.

Estas son las primeras evidencias de procesos geológicos y biológicos desarrollados durante el Paleoceno en el Uruguay.

Agradecimientos Philip Davies y Eduardo Marchesi prestaron importante colaboración comparando los endocarpos de Celtis con ejemplares actuales. Judite García proporcionó gentilmente ejemplares comparativos de gastrópodos de la Cuenca de Sao José de Itaboraí. Helena Peel corrigió el inglés del Abstract. La CSIC (Universidad de la República) financió parcialmente la realización de este trabajo.

\section{REFERENCIAS}

Beherensmeyer, A.K.; Willis, B.J \& Quade, J. 1995. Floodplains and paleosols of Pakistán Neogene and Wyoming Paleogene deposits: a comparativa study. Palaeogeography, Palaeoclimatology, Palaeoecology, 115: 37-60.

Beurlen, K. \& Sommer, F.W. 1954. Restos vegetáis fósseis e tectónica da Bacia calcárea de Itaboraí, Estado do Rio de Janeiro. DNPM/DGM. 27 p. (Boletim, 149).

Bequaert, J. 1948. Monograph of the Strophocheilidae, a neotropical family of terrestrial mollusks. Bulletin of the Museum of Comparative Zoology, Harvard, 100: 1-210.

Bossi, J.; Ferrando, L.; Fernández, A.; Elizalde, G.; Morales, H.; Ledesma, J.; Carballo, E.; Medina, E.; Ford, I. \& Montaña, J. 1975. Carta Geológica del Uruguay. Montevideo.Dir. Suelos y Fertilizantes, Ministerio de Agricultura y Pesca, 32 p., 1 mapa, Montevideo.

Bossi, J.C.\& Navarro, R. 1991. Geología del Uruguay. Montevideo. Departamento de Publicaciones, Universidad de la República, 970 p.

Cabrera, A.L. \& Zardini, E.M. 1978. Manual de la Flora de los alrededores de Buenos Aires. Buenos Aires. Acmé, 755 p.

Caorsi, J.H. \& Gofii, J.C. 1958, Geología uruguaya. Boletín del Instituto Geológico del Uruguay, 37: 1-73.

Carlisle, D. 1983. Concentration of uranium and vanadium in calóreles and gypcretes. In: WILSON, R.C. ed.: Residual Deposits. Surface related weathering processes and materials. London. Geol. Soc. London \& Blackwell. p. 185-195.

de Santa Ana, H.; Veroslavsky, G. \& González, S. 1993. Geología de los sedimentos cretácicos de las cuencas del Uruguay. Acta Geológica Leopoldensia, 40: 140-143.

Ekdale, A.A., Bromley, R.G. \& Pemberton, S.G. 1984. Ichnology. The use of trace fossils in Sedimentology and Stratigraphy. Tulsa. Society of Economic Mineralogists, Short Course Notes, 15.317 p.

Figueiras, A. \& Broggi, J. 1969. Estado actual de nuestros conocimientos sobre los moluscos fósiles del Uruguay. Parte III (Contin.). Comunicaciones de la Sociedad. Malacológica del Uruguay, 2: 333-352.

Francis, J.C. 1975. Esquema bioestratigráfico regional de la República Oriental del Uruguay. In: Congreso Argentino de Paleontología y Bioestratigrafla I, Actas ... Tucumán 1975, v. 2, p. 539-568.

Frenguelli, J. 1930. Apuntes de geología uruguaya. Boletín del Instituto de Geología y Perforaciones, 11: 1-47. Montevideo.

Frenguelli, J. 1938a. Nidi fossili di Scarabeidi e Vespidi. Societá Geológica Italiana Bolletino, 57: 77-96.

Frenguelli, J. 1938b. Bolas de escarabeidos y nidos de véspidos. Physis, 12: $348-352$.

Genise, J. \& Bown, T.M. 1994. New Miocene scarabeid and hymenopterous nests and Early Miocene (Santacrucian) paleoenvironments, Patagonian Argentina. Ichnos, 3: 107-117.

Goso, H. \& Bossi, J. 1966. Cenozoico. In: BOSSI, J. ed.: Geología del Uruguay. Montevideo. Departamento de Publicaciones, Universidad de la República, p. 259-301.

Goudie, A.S. 1983. Calcrete. In:. Goudie, A.S \& PYE, K. eds., Chemical Sediments and Geomorphology. London. Academic Press, p. 93-131.
Jones, G.H. 1956. Memoria explicativa y mapa geológico de la región oriental del Departamento de Canelones. Boletín del Instituto Geológico del Uruguay, 34: 1-193.

Klappenbach, M. A. \& Olazarri, J. 1966. Notas sobre Strophocheilidae (Molí. Gastr.) II. Aclaración del status de Strophocheilus charruanus (Frenguelli, 1930). Revista de la Facultad de Humanidaes y Ciencias, 22: $233-238$.

Klappenbach, M. A. \& Olazarri, J. 1970. Notas sobre Strophocheilidae (Molí. Gastr.) III. Eoborus, nuevo género para especies fósiles de esta familia sudamericana. ArchivfürMolluskenkunde, 100: 179-182.

Klappenbach, M. A. \& Olazarri, J. 1986. Notas sobre Strophocheilidae. IV. Eoborus berroi, nueva especie del Mioceno uruguayo. Comunicaciones Paleontológicas del Museo de Historia Natural de Montevideo, 1: 217-225.

Klein, V.C., Rodrigues Francisco, B.H. \& Souza Cunha, F.L. 1985. Resultados das pesquisas sistemáticas realizadas na Bacia de Sao José de Itaboraí, Rio de Janeiro (1972-1982). DNPM/DGM., p.653-656 ( Boletim jSérie Geología, 27, 80930 Paleontología e Estratigrafía, 2).

Lamben, R. 1939. Memoria explicativa del mapa geológico de los terrenos sedimentarios y de las rocas efusivas del Departamento de Durazno. Boletín del Instituto Geológico del Uruguay, 25b: 1-37.

Lamben, R. 1940. Memoria explicativa de un mapa geológico de reconocimiento del Departamento de Paysandú y de los alrededores de Salto. Boletín del Instituto Geológico del Uruguay, 27b: 1-39.

Lambert, R. 1941. Estado actual de nuestros conocimientos sobre la geología de la República Oriental del Uruguay. Boletín del Instituto Geológico del Uruguay, 29: 1-89.

Lima, M.R. \& Cunha, F.L.S. 1986. Análise palinológica de um nivel linhítico na Bacia de Sao José de Itaboraí, Terciario do Estado do Rio de Janeiro, Brasil. Anais Academia brasileira de Ciencias, 58: 579-588.

Magalhñes, J. 1950. Sobre a ocorréncia de Acrocarpus santosi sp. nov. no Eocénio inferior de Sao José de Itaboraí (Estado do Rio de Janeiro). Revista Científica,!: 1. Rio de Janeiro.

Méndez Alzóla, R. 1944. Geología Histórica del Uruguay. Anales del Instituto de Primaria, Época 2,7: 191-261. Montevideo.

Mones, A. 1979. Terciario del Uruguay. Síntesis geo-paleontológica. Revista de la Facultad de Humanidaes y Ciencias (serie Ciencias de la Tierra), 1: 1-27. Montevideo

Morales, H. \& Ford, 1.1988. Algunas consideraciones sobre las sedimentitas del Cretácico Superior en el Uruguay. In: ReuníOn de Geología del Uruguay I, Resúmenes Ampliados... Salto, 1988. p. 38-41

Muller, J. 1981. Fossil pollen records of extant angiosperms. Botanical Review, 47: 1-142.

Palma, J.M.C. \& Brito, I.M. 1974. Paleontología e Estratigrafía da Bacia de Sao José de Itaboraí, Estado do Rio de Janeiro. Anais Academia brasileira de Ciencias, 46: 383-406.

Parodiz, J. J. 1949. Notas sobre Strophocheilus fósiles de Argentina. Physis, 20: $180-184$

Parodiz, J. J. 1969. The tertiary non-marine Mollusca of South America. Annals of the Carnegie Museum, 40: 1-242.

Preciozzi, F.; Spoturno, J. ; Heinzen ,W. \& Rossí, P. 1985. Memoria explicativa de la Carta Geológica del Uruguay a la escala 1:500.000. Montevideo. DINAMIGE. 90 p. 
Retallack, G. 1984a. Completeness of the rock and fossil record: some estímales using fossil soils. Paleobiology, 10: 59-78.

Retallack, G. 1984b. Trace fossils of burrowing beetles and bees in an Oligocene paleosol, Badlands National Park, South Dakota. Journal of Paleontology, 58: 571-592.

Retallack, G. 1990. Soils of the past. An introduction lo paleopedology. London. Harper Collins, $520 \mathrm{p}$.

Rodrigues Francisco, B.H. \& Cunha, F.L. S. 1978. Geología e Estratigafia de Sao José, Municipio de Itaboraí, RJ. Anais Academia brasileira de Ciencias, 50:381-416.

Serra, N. 1945. Memoria explicativa del mapa geológico del Departamento de Soriano. Boletín del Instituto Geológico del Uruguay, 32: 1-42.

Sprechmann, P.; Bossi, J. \& da silva, M. 1981. Cuencas del Jurásico y Cretácico del Uruguay. In: Volkheimer, W.; Mussachio, E.A., Eds. Cuencas Sedimentarias del Jurásico y Cretácico de América del Sur. Buenos Aires.Comité del Jurásico y Cretácico de América del Sur. p. $239-270$.
Thackray.G.D. 1994. Fossil nests of sweat bees (Halictinae) from a Miocene paleosol, Rusinga Island, Western Kenya. Journal of Paleontology, 68: 795-800.

Veroslavsky, G.; Martínez, S. \& de Santa Ana, H.. 1997. Calórelas de aguas subterráneas: génesis de los depósitos carbonáticos de la Cuenca de Santa Lucía (Sur del Uruguay, Cretácico Superior- Paleógeno). Revista de la Asociación Argentina de Sedimentología, 4: 25-35.

Walther, K. 1931. Sedimentos gelíticos y clastogelíticos del Cretáceo Superior y Terciario uruguayos. Boletín del Instituto de Geología y Perforaciones, 13: 1-142. Montevideo.

Wright, V.P. \& Tucker, M.E. 1991. Calcretes: an introduction. In: Wright, V.P \& Tucker, M.E. eds.: Calcretes. International Association of Sedimentologists, Reprint Series, 2. p.1-22.

MANUSCRITO A-920

Manuscrito recebido em 17 de junho de 1997 Revisáo dos autores em 01 de outubro de 1997 Revisáo aceita em 05 de outubro de 1997 